\title{
Investigating low energy QCD with kaonic atoms: the SIDDHARTA experiment at DA $\Phi$ NE
}

\author{
A.Scordo ${ }^{a *}$ \\ M. Bazzi ${ }^{a}$, G. Beer ${ }^{b}$, L. Bombellic , A.M. Bragadireanu ${ }^{a, d}$, M. Cargnelli ${ }^{e}$, A. Clozza $^{a}$, \\ C. Curceanu $\left(\right.$ Petrascu) ${ }^{a}$, A. d'Uffizi ${ }^{a}$, C. Fiorini ${ }^{c}$, T. Frizzi ${ }^{c}$, F. Ghio $^{f}$, B. Girolami ${ }^{f}$, \\ C. Guaraldo ${ }^{a}$, R.S. Hayano ${ }^{g}$, M. Iliescu ${ }^{a, d}$, T. Ishiwatari ${ }^{e}$, M. Iwasaki ${ }^{h}$, P. Kienle ${ }^{e, i}$, P. Levi

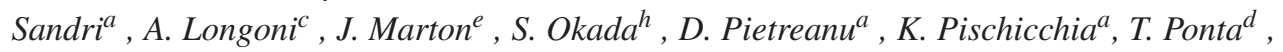 \\ A. Rizzo ${ }^{a}$, A. Romero Vidal ${ }^{l}$, E. Sbardella ${ }^{a}$ H. Shi ${ }^{g}$, D.L. Sirghi ${ }^{a, d}$, F. Sirghi ${ }^{a, d}$, H. Tatsuno ${ }^{a}$, \\ A. Tudorache ${ }^{d}$, V. Tudorache ${ }^{d}$, O. Vazquez Doce ${ }^{m}$, E. Widmann ${ }^{e}$, B. Wünschek ${ }^{e}$, J. Zmeskal \\ ${ }^{a}$ INFN, Laboratori Nazionali di Frascati, Frascati (Roma), Italy \\ ${ }^{b}$ Dep. of Phys. and Astro., Univ. of Victoria, Victoria B.C., Canada \\ ${ }^{c}$ Politecnico di Milano, Sez. di Elettronica, Milano, Italy \\ ${ }^{d}$ IFIN-HH, Magurele, Bucharest, Romania \\ e Stefan-Meyer-Institut für subatomare Physik, Vienna, Austria \\ ${ }^{f}$ INFN Sez. di Roma I and Inst. Superiore di Sanita, Roma, Italy \\ ${ }^{g}$ Univ. of Tokyo, Tokyo, Japan \\ ${ }^{h}$ RIKEN, The Inst. of Phys. and Chem. Research, Saitama, Japan \\ ${ }^{i}$ Tech. Univ. München, Physik Dep., Garching, Germany \\ ${ }^{l}$ Universidade de Santiago de Compostela, Santiago de Compostela, Spain \\ ${ }^{m}$ Excellence Cluster Universe, Technische Universität München, Garching, Germany \\ E-mail: alessandro.scordodlnf.infn.it
}

\begin{abstract}
Kaonic atoms measurements offer the key to unlock secrets of the low-energy QCD in the strangeness sector, a field still largely unexplored. Their experimental studies allow to access fundamental information, such as the antikaon-nucleon isospin dependent scattering lengths which are, in turn, vital information for understanding the mechanism of chiral symmetry breaking. In the past years, the studies of kaonic atoms received a big boost with the advent of new experimental techniques, as those pioneered by SIDDHARTA, based on the use of fast Silicon Drift Detectors for precision X-ray spectroscopy and of the ideal kaon beam offered by DAФNE. The precision achieved by SIDDHARTA at DAФNE in measuring kaonic helium 3 and 4 and kaonic hydrogen is unique. In this work we'll present the results of the measurement of the strong interaction induced shift and width of kaonic hydrogen 1s level, and the shift measurement of the 2 p level for kaonic helium 3 (first measurement ever) and 4.
\end{abstract}

50th International Winter Meeting on Nuclear Physics - Bormio2012,

23-27 January 2012

Bormio, Italy

${ }^{*}$ Speaker. 


\section{Contents}

1. Introduction 2

2. Low $\mathrm{Z}$ kaonic atoms puzzles 3

2.1 Kaonic hydrogen puzzle 3

2.2 Kaonic Helium Puzzle 3

3. The SIDDHARTA experimental setup 4

4. Data analysis 5

4.1 Calibration 5

4.2 Kaonic atoms spectra 5

5. Results 6

5.1 Kaonic hydrogen 6

5.2 Kaonic helium 8

6. Conclusions $\quad 8$

\section{Introduction}

The strong interaction, described by the QCD in the framework of the Standard Model, is still hiding many mysteries, especially in the low-energy limit, the so called non-perturbative regime. Low energy phenomena in strong interactions are described by effective field theories, which contain appropriate degrees of freedom to describe physical phenomena occurring at the nucleonmeson scale. Chiral perturbation theory was extremely successful in describing systems like pionic atoms, however it is not directly applicable for the kaonic systems, where non-perturbative coupledchannel techniques can be used ([1]); these theories are still waiting to be verified by experimental data. With the advance of the experimental techniques, both in the accelerator and detector sectors, we are presently able to perform very high precision measurements, which will bring to a deeper and more complete understanding of the many open questions in the QCD. Among these techniques which saw a huge progress in the last decade, a special place is hold by the measurements of X-rays emitted by exotic atoms. Exotic atoms are QED bound systems, that means any system in which a negatively charged particle, other than an electron, orbits a nucleus, under conditions in which the principal interaction with the nucleus is electromagnetic. For example the electron might be replaced by a negative muon, pion, kaon, sigma, or by an antiproton. Exotic atoms formed with the muons are subject to the weak interaction, while for all other named exotic atoms the strong interaction is involved in addition to the electromagnetic one with the nucleus. The electromagnetic interaction with the nucleus is very well known, therefore, even a small deviation from the 
electromagnetic value e.g. due to strong interaction, could be measured by performing precision experiments. Among the exotic atoms, the kaonic ones, having a $K^{-}$replacing an $e^{-}$orbiting the nucleus, play a special role due to the strange quark contained in the kaon. With the advent of clean kaon beams, as the one provided by the DAФNE collider, and of performant, fast, X-ray detectors as the Silicon Drift Detectors, the kaonic atoms studies entered a new era.

\section{Low $\mathrm{Z}$ kaonic atoms puzzles}

In order to improve our knowledge on the QCD in the low-energy limit, in particular in the strangeness sector, a special role is played by the low $\mathrm{Z}(\leq 2)$ kaonic atoms, i.e. kaonic hydrogen, helium and deuterium. Kaonic deuterium has never been measured, while both $\mathrm{KH}$ and $\mathrm{KHe}$ were the protagonists of two longstanding puzzles, consisting in a discrepancy between theoretical predictions and experimental data. These two puzzles have been definitively solved by the SIDDHARTA experiment, which performed the most precise measurements on both $K H \varepsilon_{1 s}$ [2] and $K^{3,4} \mathrm{He} \varepsilon_{2 s}$ [3].

\subsection{Kaonic hydrogen puzzle}

Historically there were several measurements of the strong-interaction shift $\varepsilon_{1 s}$ and width $\Gamma_{1 s}$ of kaonic hydrogen $([4,5,6,7,8])$. In the 1970s and the 1980s three groups ([4, 5, 6]) reported a measured attractive shift (positive $\varepsilon_{1 s}$ ), while the information extracted from the analyses of the low energy $\mathrm{KN}$ data $([9,10,11])$ shows a repulsive shift (negative $\varepsilon_{1 s}$ ). This contradiction has been known as the "kaonic hydrogen puzzle". In 1997, the first distinct peaks of the kaonichydrogen X rays were observed by the KEK-PS E228 group [7] with a significant improvement in the signal-to-background ratio by the use of a gaseous hydrogen target, where previous experiments had employed liquid hydrogen. It was crucial to use a low-density target, namely a gaseous target, because the X-ray yields quickly decrease towards higher density due to the Stark mixing effect. The observed repulsive shift was consistent in sign with the analysis of the low energy KN scattering data, resolving the long-standing discrepancy. More recent values reported by the DEAR group in 2005 [8], with substantially reduced errors, firmly established the repulsive shift obtained in the previous E228 experiment.

\subsection{Kaonic Helium Puzzle}

For what concerns the kaonic helium, its transition to 1s level couldn't be observed due to the very low yield. Before the SIDDHARTA experiment, the situation was rather ambiguous. There existed only four experiments: three [12, 13, 14], performed more than 20 years ago, giving, within few $\sigma \mathrm{s}$, results which are more than an order of magnitude higher with respect to the theoretical predictions and a more recent one, performed at KEK [15], which was, instead, compatible with theoretical predictions $([16,17])$, but incompatible with the previous experiments. The average value of the first three experiments was $\varepsilon_{2 p}=-43 \pm 8 \mathrm{eV}$ which was more than three standard deviations different with respect to the last one; the situation is shown in fig. 1.

A conclusive precise measurement on $K^{4} \mathrm{He}$ was then needed in order to close the "puzzle". Moreover, a study including the first measurement of $K^{3} \mathrm{He}$ could yield invaluable informations on the $K-p$ and the $K-n$ interactions [18]. 


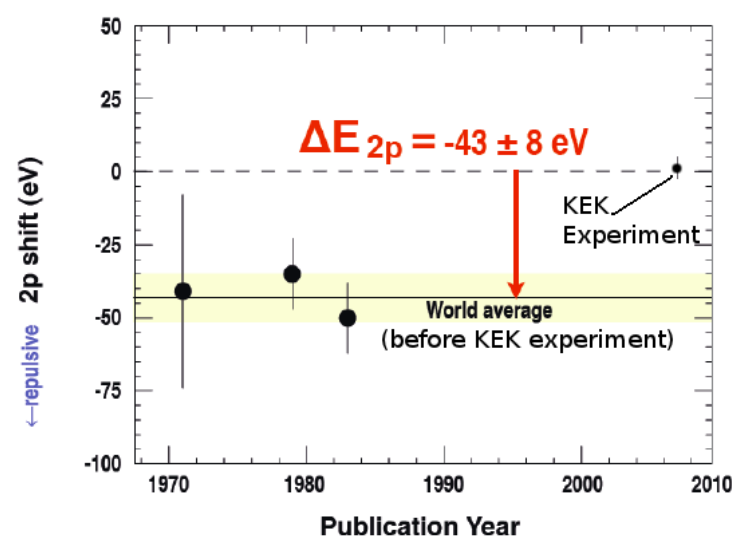

Figure 1: Summary of experimental results on kaonic helium 4

\section{The SIDDHARTA experimental setup}

SIDDHARTA (SIlicon Drift Detector for Hadronic Atoms Research by Timing Application) $[19,20,21]$ is a high precision experiment dedicated to the study of kaonic atoms, on the DAФNE collider at Laboratori Nazionali di Frascati of INFN. In fig. 2, a schematic view of the setup is shown.

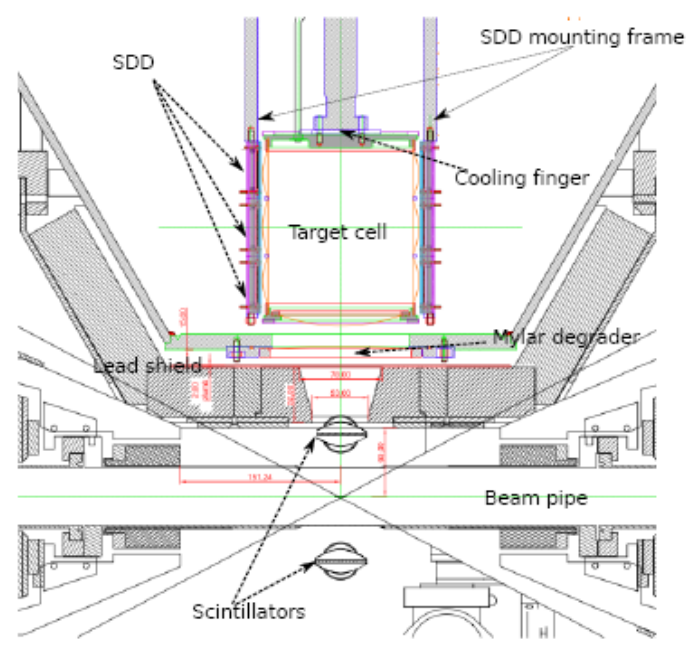

Figure 2: Schematic and side view of the SIDDHARTA setup in the interaction region

Electrons and positrons collide in the interaction region (IR), where a cylindrical beam pipe with a diameter of $6 \mathrm{~cm}$ and $350 \mu \mathrm{m}$ wall thickness is placed (AL6082 [22]). The $\Phi$ mesons which are produced in the IR, decay into back-to-back $K^{+}-K^{-}$pairs with a branching ratio of $49.2 \%$. The kaons coming out from the interaction region are detected by two scintillators (BC$420,152 \times 72 \mathrm{~mm}, 1.5 \mathrm{~mm}$ thickness), placed above and below the beam pipe and read out at both sides by Hamamatsu PMTs (Hamamatsu R4998). The coincidence between the two scintillators (4 PMTs) is used as trigger for the data acquisition. In order to slow down the kaons to a velocity compatible with their capture in the atomic orbits, a mylar degrader, ad hoc shaped taking into 
account the small boost $\Delta \Phi$ in the radial direction caused by a small crossing angle between $e^{+}$and $e^{-}$beams, is placed just before the entrance window of the target cell. Degrader dimensions are $120 \times 120 \mathrm{~mm}$, with an increasing thickness (according to the boost direction) from 100 to $800 \mu \mathrm{m}$. Kaons enter in the target cell, which is a cylindrical kapton chamber of $16.5 \mathrm{~cm}$ height, $7 \mathrm{~cm}$ radius and $75 \mu \mathrm{m}$ thickness. The target is filled with gas (hydrogen, helium or deuterium) at cryogenic temperature. The X-rays emitted from kaonic helium atoms are then detected by Silicon Drift Detectors [20], placed all around the target cell and cooled down to $170 \mathrm{~K}$. Detectors are read out by an ad hoc built read out electronics [21,23], and are arranged in arrays of 3 detectors each.

\section{Data analysis}

\subsection{Calibration}

The Silicon Drift Detectors used by the SIDDHARTA experiment and developed in the framework of the collaboration, were used for the first time on a collider. In order to obtain a precision of $0.1 \%$ on the peak positions, a precise calibration of each SDD is needed; each 15 production runs, a calibration run (in the same beam conditions as in the standard production runs) has been taken. Calibration was performed using an X-ray tube, located below the interaction region and aligned with the entrance window of the target cell, activating $\mathrm{Ti}$ and $\mathrm{Cu}$ foils placed inside the vacuum chamber. Characteristic X-ray lines of $\mathrm{Ti}$ and $\mathrm{Cu}$ have been then used for the calibration of the detectors. A typical calibration spectrum is shown in fig. 3.
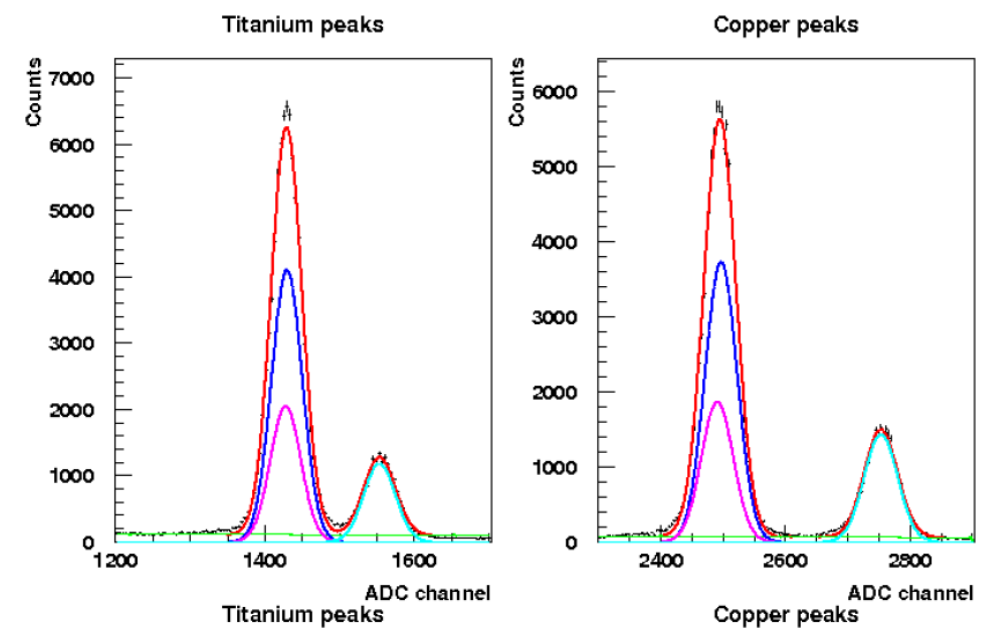

Figure 3: Typical calibration spectra for Ti and $\mathrm{Cu}$; total fit (red), $K_{\alpha}$ (blue) and $K_{\beta}$ (light blue) are plotted. Note that the fluorescence $K_{\alpha}$ lines consist of $K_{\alpha 1}$ and $K_{\alpha 2}$ lines, both of which are shown.

\subsection{Kaonic atoms spectra}

In order to obtain the best kaonic atoms spectra, some cuts and selections have to be applied to the raw data:

- SDD selection: only the SDD having a good behaviour and working properly are selected. 
- Drift time selection: using timing information coming from the Kaon Monitor scintillators, events triggered only by kaons can be identified. Drift time represents the time difference between a trigger from the Kaon Monitor and the hit on a SDD. Kaons related events are all lying under a peak and can be then selected (see fig. 4).

- Kaons identification: under the drift time peak, also events of background, mostly generated by $e^{-}$and $e^{+}$lost from circulating beams and correlated in time with the kaons production, are present. These can be separated from the kaons using the time of flight information, as shown in fig. 5.

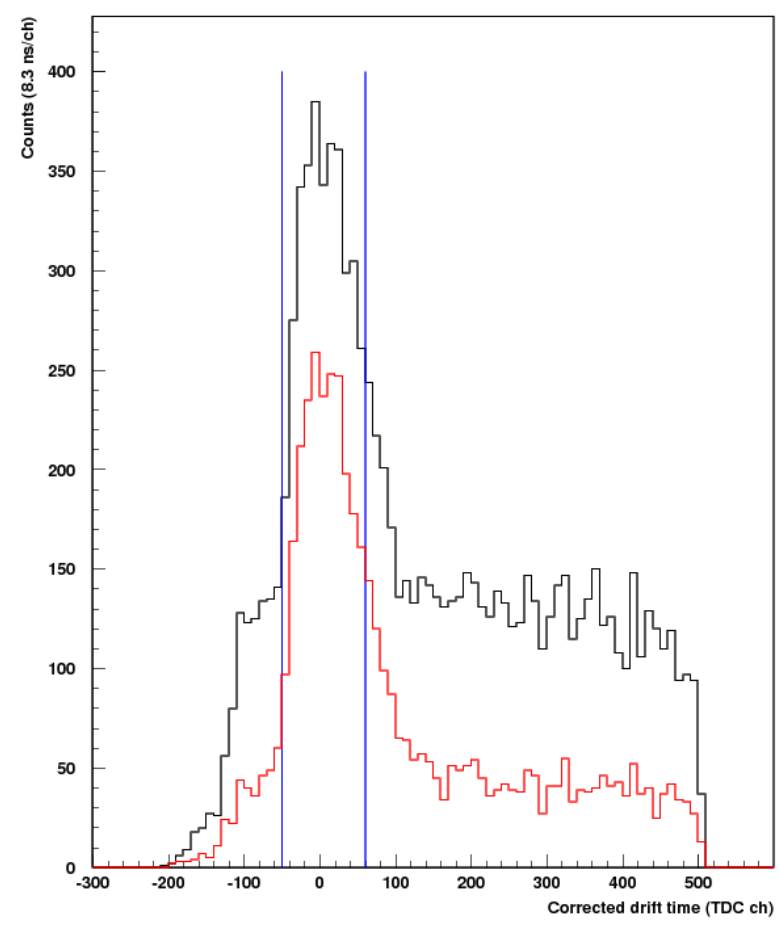

Figure 4: Drift time spectrum with kaons' peak selection evidenced, before (black) and after (red) the kaons identification cut.

\section{Results}

\subsection{Kaonic hydrogen}

The final kaonic hydrogen and deuterium X-ray energy spectra are shown in fig. 6.

The K-series X-rays of kaonic hydrogen were clearly observed while those for kaonic deuterium were not visible. This appears to be consistent with the theoretical expectation of lower $\mathrm{X}$-ray yield and greater transition width for deuterium ([24]) than for kaonic hydrogen. However, the kaonic deuterium spectrum can be used to characterize the background. The vertical dot-dashed line in fig.6 indicates the X-ray energy of kaonic-hydrogen $K_{\alpha}$ calculated using only the electromagnetic interaction (EM). Comparing the kaonic-hydrogen $K_{\alpha}$ peak and the EM value, a repulsive 


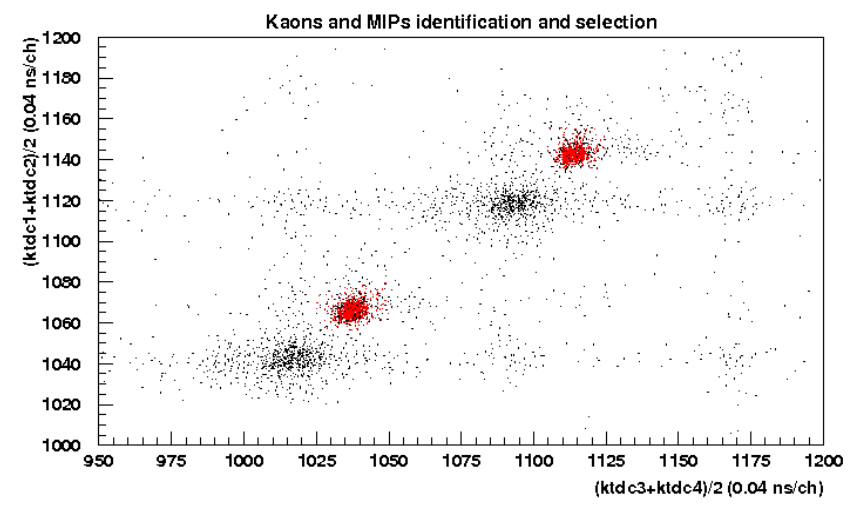

Figure 5: 2d plot of Kaon Monitor TDCs shownig clusters related to kaons (red) and MIPs (black).

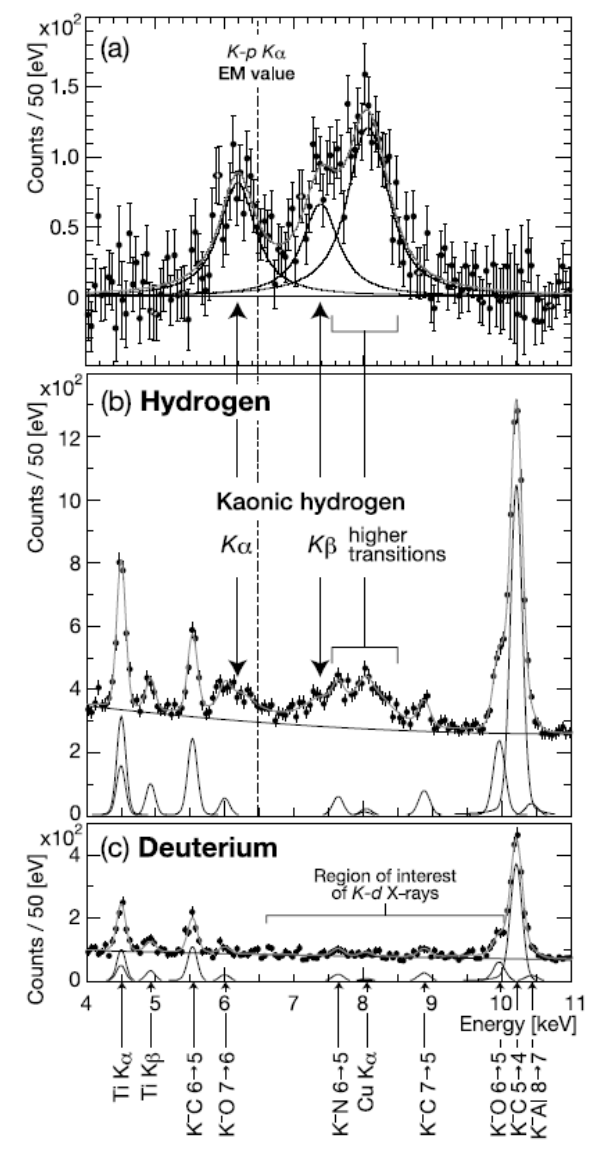

Figure 6: A global simultaneous fit result of the X-ray energy spectra of hydrogen and deuterium data. (a) Residuals of the measured kaonic-hydrogen X-ray spectrum after subtraction of the fitted background, clearly displaying the kaonic-hydrogen $\mathrm{K}$-series transitions. The fit components of the K-p transitions are also shown, where the sum of the function is drawn for the higher transitions (greater than $K_{\beta}$ ). (b), (c) Measured energy spectra with the fit lines. Fit components of the background X-ray lines and a continuous background are also shown. The dot-dashed vertical line indicates the EM value of the kaonic-hydrogen $K_{\alpha}$ energy. (Note that the fluorescence $K_{\alpha}$ line consists of $K_{\alpha 1}$ and $K_{\alpha 2}$ lines, both of which are shown.) 
shift of the kaonic-hydrogen 1s-energy level is easily seen. Many other lines from kaonic-atom $\mathrm{X}$-rays were detected in both spectra as indicated with arrows in the figure. These kaonic-atom lines result from high-n X-ray transitions of kaons stopped in the target-cell wall made of kapton $\left(\mathrm{C}_{22} \mathrm{H}_{10} \mathrm{O}_{5} \mathrm{~N}_{2}\right)$ and its support frames made of aluminium. There are also characteristic X-rays from titanium and copper foils installed for X-ray energy calibration. A global simultaneous fit of the hydrogen and deuterium spectra has been performed, whose results are shown in Fig. 6 (b) and (c). The kaonic-hydrogen lines were represented by Lorentz functions convoluted with the detector response function, where the Lorentz width corresponds to the strong-interaction broadening of the 1s state. The region of interest of K-d X-rays is illustrated in Fig. 6 (c). The 1s-level shift $\varepsilon_{1 s}$ and width $\Gamma_{1 s}$ of kaonic hydrogen were determined to be [2]:

$$
\begin{gathered}
\varepsilon_{1 s}=283 \pm 36(\text { stat }) \pm 6(\text { syst }) \mathrm{eV} \\
\Gamma_{1 s}=541 \pm 89(\text { stat }) \pm 22(\text { syst }) \mathrm{eV}
\end{gathered}
$$

The quoted systematic error is a quadratic summation of the contributions from the systematic errors on the SDD gain shift, the SDD response function, the ADC linearity, the low energy tail of the kaonic-hydrogen higher transitions, the energy resolution, and the procedural dependence shown by independent analysis.

\subsection{Kaonic helium}

In the case of kaonic helium, an identical procedures for calibration and data analysis was used both for $\mathrm{K}^{3} \mathrm{He}$ and $\mathrm{K}^{4} \mathrm{He}$. The obtained spectra are shown in fig. 7

In the upper picture, the peak seen at $6.2 \mathrm{keV}$ is identified as the $K^{3} \mathrm{He} L_{\alpha}$ line (the $3 d \rightarrow 2 p$ transition). In the lower picture, the peak seen at $6.4 \mathrm{keV}$ is identified as the $K^{4} H e L_{\alpha}$ line. In addition to these lines, small other peaks are clearly visible which identification has been already explained in the previous section. The strong-interaction shifts of the kaonic helium $2 p$ states are obtained from the difference between the experimentally determined values and the QED calculated ones $[25,26]$. The results are:

$$
\begin{aligned}
& \varepsilon_{2 p}\left(K^{3} \mathrm{He}\right)=2 \pm 2(\text { stat }) \pm 4(\text { syst }) \mathrm{eV} \\
& \varepsilon_{2 p}\left(K^{4} \mathrm{He}\right)=5 \pm 5(\text { stat }) \pm 4(\text { syst }) \mathrm{eV}
\end{aligned}
$$

where the second terms denoted as (stat) are the statistical errors and the third terms denoted as (syst) are the systematic errors. The helium 4 result is in agreement, within the errors, with the results reported by the E570 [15] and SIDDHARTA [3] collaborations, while is not in agreement with the eldest measurements $([12,13,14])$. This is probably due to the fact that, in those experiments, the helium target was a liquid one in which, as indeed was done in the KEK experiment ([15]), a big Compton effect is present and has to be taken into account in the analysis procedure.

\section{Conclusions}

The SIDDHARTA collaboration performed the most precise measurement on kaonic hydrogen, which is presently being considered by various theoretical analysis ([27, 28, 29]). Moreover, 

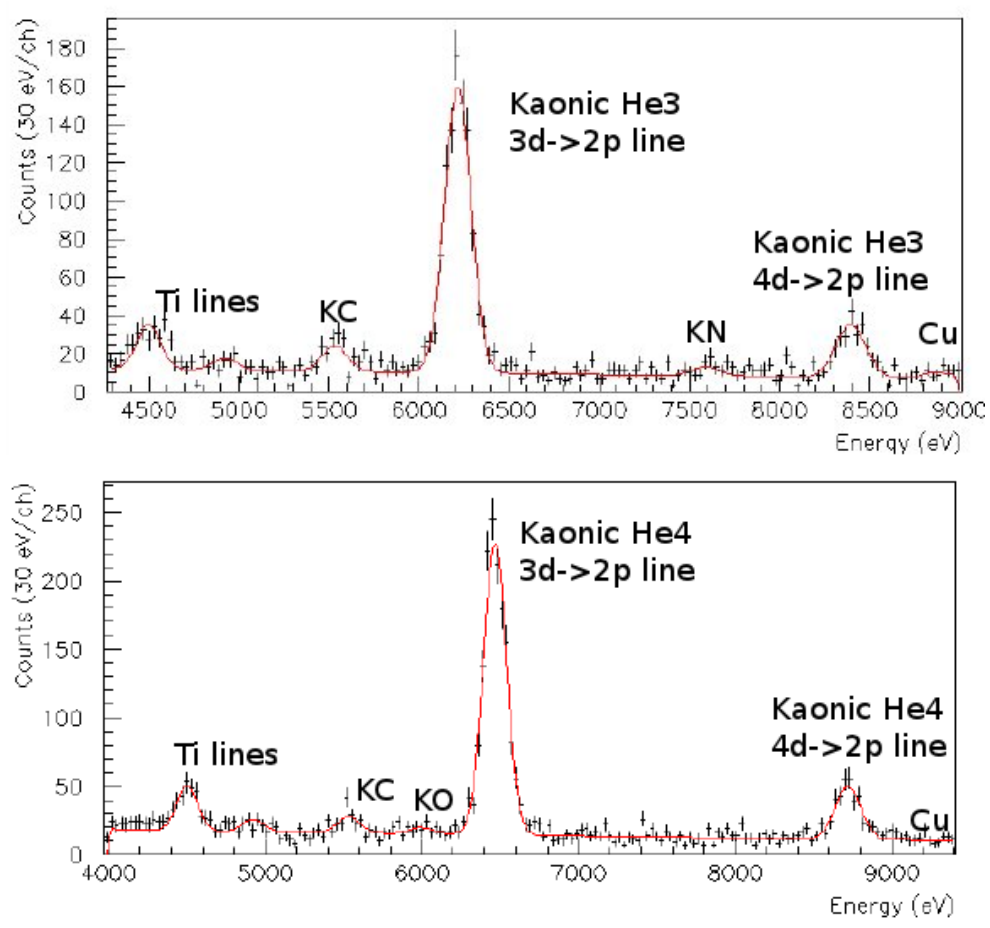

Figure 7: Fitted spectra of the kaonic ${ }^{3} \mathrm{He}$ (top) and ${ }^{4} \mathrm{He}$ (bottom) X-rays. The $3 d \rightarrow 2 p$ transitions are seen around $6 \mathrm{keV}$. Together with these peaks, small others are seen, which are the kaonic atom X-ray lines produced by kaons stopping in the target window made of Kapton, and the Ti and $\mathrm{Cu}$ lines .

SIDDHARTA measured for the first time ever the kaonic helium 3 transitions to the $2 p$ level and the first, similar, measurement, of kaonic helium 4 with a gaseous target. Presently, plans for an upgrade of the experimental setup (SIDDHARTA-2,[30]) for an enriched program for the near future are undergoing, with the main aim to perform the first measurement ever on kaonic deuterium and other exotic atoms studies.

\section{Acknowledgements}

We thank C. Capoccia, G. Corradi, B. Dulach and D. Tagnani from LNF-INFN; and H. Schneider, L. Stohwasser, and D. Stückler from Stefan-Meyer-Institute, for their fundamental contriburion in designing and building the SIDDHARTA setup. We thank as well the DAФNE staff for the excellent working conditions and permanent support. Part od this work was supported by HadronPhysics I3 FP6 European Community program, Contract No. RII3-CT-2004-506078; the European Community-Research infrastructure Integrating Activity "Study of Strongly Interacting Matter" (HadronPhysics2, Grant Agreement No. 227431), and HadronPhysics 3 (HP3), Contract No. 283286 under the Seventh Framework Programme of EU; Austrian Federal Ministry of Science and Research BMBWK 650962/0001 VI/2/2009; Romanian National Authority for Scientific Research, Contract No. 2-CeX 06-11-11/2006; the Grant-in-Ais for Specially Promoted Research (20002003), MEXT, Japan; the Austrian Science Fund (FWF): [P20651-N20]; and the DFG Excellence Cluster Universe of the Technische Universität München. 


\section{References}

[1] J. Caro-Ramon, et al., Nucl. Phys. A 672 (2000) 249-269.

[2] M. Bazzi, et al., Phys. Lett. B 704 (2011) 113-117.

[3] M. Bazzi, et al., Phys.Lett.B 697 (2011) 199-202.

[4] J. D. Davies, et al., Phys. Lett. B 83 (1979) 55.

[5] M. Izycki, et al., Z. Phys. A 297 (1980) 11.

[6] P. M. Bird, et al., Nucl. Phys. A 404 (1983) 482.

[7] M. Iwasaki, et al. Phys. Rev. Lett. 78 (1997) 3067; T. M. Ito, et al., Phys. Rev. C 58 (1998) 2366.

[8] G. Beer, et al. (DEAR Collaboration), Phys. Rev. Lett. 94 (2005) 212302.

[9] W.E. Humphrey and R.R. Rose, Phys. Rev. 127 (1962) 1305.

[10] J.K. Kim, Phys. Rev. Lett. 19 (1967) 1074.

[11] A.D. Martin, Nucl. Phys. B 179 (1981) 33.

[12] C.E. Wiegand and R.H. Pehl, Phys. Rev. Lett 27 (1971) 1410.

[13] C.J. Batty et al., Nucl. Phys. A 326 (1979) 455.

[14] S. Baird et al., Nucl. Phys. A 392 (1983) 297.

[15] S. Okada et al., Phys. Lett. B 653 (2007) 387.

[16] C.J. Batty, Nucl. Phys. A 508 (1990) 89c.

[17] R. Seki, Phys. Rev. C 5 (1972) 1196.

[18] Y. Akaishi, in: Proc. Int. Conf. on Exotic Atoms (EXA05), Austrian Academy of

[19] J. Zmeskal, in: A. Hirtl, J. Marton, E.Widmann, J. Zmeskal (Eds.), Proceedings EXA05, Austrian Academy of Science Press, Vienna, 2005,p. 139.

[20] J. Zmeskal, SIDDHARTA Technical Note IR-2 (2003), LNF-INFN

[21] C. Curceanu, SIDDHARTA Technical Note IR-3 (2003), LNF-INFN

[22] C. Milardi, DAФNE interaction regions upgrade arXiv:0803.1450v1.

[23] T. Frizzi, Detectors and Electronics for Hadronic Physics Experiments, Doctoral Dissertation, Politecnico di Milano (2007).

[24] T. Koike, T. Harada, Y. Akaishi, Phys. Rev. C 53 (1996) 79

[25] V. Lucherini, et al., Nucl. Instr. Meth. A 496 (2003) 315.

[26] S.G. Karshenboim, et al., Can. J. Phys. 84 (2006) 107. Sciences Press, Vienna, 2005, p. 45, http://dx.doi.org/10.1553/exa05s45.

[27] M. Doring, et al., Phys.Lett. B 704 (2011) 663-666.

[28] Yoichi Ikeda, et al., Phys. Lett. B 706 (2011) 63-67.

[29] E. Friedman, A. Gal, arXiv:1201.3770 [nucl-th]

[30] SIDDHARTA-2 proposal The upgrade of the SIDDHARTA apparatus for an enriched scientific case, 14/06/2010, Presented to the LNF International Scientific Committee, 24 June 2010 